\title{
Monitoring Cleaning-In-Place by Electrical Resistance Tomography with Dynamic References
}

DOI:

10.1109/IST.2016.7738241

\section{Document Version}

Accepted author manuscript

Link to publication record in Manchester Research Explorer

\section{Citation for published version (APA):}

Wang, S., \& Yin, W. (2016). Monitoring Cleaning-In-Place by Electrical Resistance Tomography with Dynamic References. In IEEE Instrumentation \& Measurement Society IEEE. https://doi.org/10.1109/IST.2016.7738241

\section{Published in:}

IEEE Instrumentation \& Measurement Society

\section{Citing this paper}

Please note that where the full-text provided on Manchester Research Explorer is the Author Accepted Manuscript or Proof version this may differ from the final Published version. If citing, it is advised that you check and use the publisher's definitive version.

\section{General rights}

Copyright and moral rights for the publications made accessible in the Research Explorer are retained by the authors and/or other copyright owners and it is a condition of accessing publications that users recognise and abide by the legal requirements associated with these rights.

\section{Takedown policy}

If you believe that this document breaches copyright please refer to the University of Manchester's Takedown Procedures [http://man.ac.uk/04Y6Bo] or contact uml.scholarlycommunications@manchester.ac.uk providing relevant details, so we can investigate your claim.

\section{OPEN ACCESS}




\title{
Monitoring Cleaning-In-Place by Electrical Resistance Tomography with Dynamic References
}

\author{
Shupei Wang; Wuliang Yin \\ School of Electrical and Electronic Engineering \\ University of Manchester \\ Manchester, UK \\ shupei.wang@postgrad.manchester.ac.uk \\ Wuliang.Yin@manchester.ac.uk
}

\begin{abstract}
In multifunctional food and detergent production lines, accurate identification of ending point of the cleaning process for the previous product is crucial to ensure product integrity. In this paper, a commercial electrical resistance tomography system was applied to monitoring a lab Cleaning-In-Place circuit. Several image reconstruction algorithms are compared for the accurate identification of the end point and an optimized algorithm based on Tikhonov regularization with dynamic references is proposed and verified to be the best solution. The capabilities of this novel approach are discussed.
\end{abstract}

Keywords-Clean-In-Place(CIP); Electrical Resistance Tomography(ERT); Tikhonov regularization; dynamic reference; ending point; image reconstruction

\section{INTRODUCTION}

Clean-In-Place(CIP), is an important industrial process in food and detergent plants. It aims at the removal/cleaning of soil inside the production line, which can endanger the process sterility, without dismantling the plant[1]. It has always been a crucial topic, especially for plants with multifunctional production lines, to monitor and analyze the process of CIP. Electrolyte-insulator-semiconductor (EIS) $\mathrm{pH}$ sensor has been adopted by researcher to judge the completion of CIP process. However, this method can only monitor the flow near the surface of the semiconductor which might lead to false reading of the completion stage of the whole process[2]. Several ultrasonic acoustic methods for fouling detection were also tried, but the cost of sophisticated instrumentation limits the possibility of pervasive applications in real industry[3,4].

Electrical Resistance Tomography (ERT), is a nonintrusive technology which has been widely adopted in industrial process monitoring[5]. It involves the acquisition of boundary voltages from sensors(electrodes) located on the periphery of an object, such as a process vessel, pipeline, etc. $[5,6]$. The boundary voltages vary with the change of external electric field (normally triggered by the injection of current), and the latter is caused by the change of conductivity distribution on the electrode plane[7]. With inverse calculation algorithms, the boundary voltages can be computed into reconstructed cross-sectional images of the object. Because of its rapid measurement speed, imaging ability and relatively low cost, ERT has become a popular technique in industrial process monitoring and analysis.

In the present paper, ERT is used as a new approach to analyze the comprehensive spatial and time-varying conductivity changes during a CIP process, so as to locate the most difficult point to be cleaned in the circuit and also the ending point of the whole cleaning process. Section II describes the basic theory and concepts of ERT and the choice of algorithms, followed by the optimization applied to the basic algorithms. Section III introduces the instruments and procedures adopted in the experiment and also the principle in analyzing the results. Then preliminary results generated by two conventional algorithms are presented and compared with those from an optimized algorithm. The last section concludes the capabilities and drawbacks of this new approach.

\section{Methodology}

\section{A. Sensitivity and forwards problem}

The basic theorem of sensitivity in Electrical Impedance Tomography can be presented as follows[8]:

$$
S_{j, k}=-\int \vec{E}_{\varphi} \cdot \vec{E}_{\psi} d a \approx-\vec{E}_{\varphi} \cdot \vec{E}_{\psi}
$$

$S_{j, k}$ is the sensitivity coefficient that relates the changes in coupling for a particular exciter and detector pair to the change in the electromagnetic properties of a small perturbation within the detection range of the pair [8]. $k$ stands for pixel number in the reconstructed image. $j$ stands for voltage projection formed by 2 electrode pairs. $\vec{E}_{\varphi}$ and $\vec{E}_{\psi}$ are the electric field strength in the corresponding pixel when either of the electrode pairs is acting as the exciter injecting current into the field, while the other electrode pair is acting as the detector.

The core equation of the forward problem based on the sensitivity theorem is in [9].

$$
\frac{\Delta V_{j}}{V_{R, j}} \approx-\frac{\sum_{k=1}^{W} \Delta \sigma_{k} \cdot s_{j, k}}{\sum_{k=1}^{W} \sigma_{R, k} \cdot s_{j, k}}
$$

where $w$ is the total number of pixels in the reconstructed image; $s_{j, k}$ is the sensitivity of $k$ th pixel in projection $j ; V_{R, j}$ and $\sigma_{R, k}$ are the reference voltage in projection $j$ and the reference conductivity of pixel $k$ respectively; $\Delta V_{j}$ and $\Delta \sigma_{k}$ 
are the voltage change in projection $j$ and the conductivity change of pixel $k$.

This explains how changes in the conductivity distribution lead to corresponding changes in the measured boundary voltages. Furthermore, the equation can be derived as:

$$
\bar{V}_{p * 1}=-\bar{J}_{p * w} \bar{\sigma}_{w * 1}
$$

where $p$ is the total number of projections, $\bar{J}_{p * w}$ is the normalized sensitivity matrix where each sensitivity value is divided by the sum of all the sensitivity values under each projection, $\bar{V}_{p * 1}$ is the voltage change vector,

$$
\bar{V}_{p * 1}=\left[\frac{\Delta V_{1}}{V_{R 1}}, \frac{\Delta V_{2}}{V_{R 2}} \cdots \frac{\Delta V_{p}}{V_{R p}}\right]^{-1}
$$

$\bar{\sigma}_{w * 1}$ is the conductivity change vector,

$$
\bar{\sigma}_{w * 1}=\left[\frac{\Delta \sigma_{1}}{\sigma_{R}}, \frac{\Delta \sigma_{2}}{\sigma_{R}} \ldots \frac{\Delta \sigma_{w}}{\sigma_{R}}\right]^{-1}
$$

\section{B. Inverse Problem}

The basic idea of inverse problem in ERT is to reveal the relationship between changes of conductivity distribution in the field and measured boundary voltages. Mathematically, this process is the inverse of equation (5):

$$
\bar{\sigma}_{w * 1}=-\bar{J}_{w * p}^{-1} \bar{V}_{p * 1}
$$

Due to the soft field nature of electrical tomography, this is a severely ill-posed inverse problem because of the large condition number of sensitivity matrix (normally $10^{6}$ ) and insufficient number of independent measurements (less than the number of pixels)[10]. In practice it means for any given measurement precision, there are arbitrarily large changes in the conductivity distribution which are undetectable by boundary voltage measurements at that precision[11]. Hence algorithms need to be adopted to minimize errors in the inverse calculations.

\section{Choice of Algorithm}

\section{1) Linear Back Projection(LBP)}

LBP is one of the first algorithms adopted in the attempt to reconstruct images for the electrical tomography. It is less accurate but with rapid response speed for computing because of its simplicity. The basic idea is to consider the sensitivity matrix $\bar{J}$ as a linear mapping from conductivity vector space to boundary voltage vector space (which is not true for the soft field domain), so the transposed sensitivity matrix $\vec{J}^{T}$ can be considered as the related mapping from boundary voltage vector space to conductivity vector space[12]. Hence,

$$
\bar{\sigma}=\bar{J}^{T} \bar{V}
$$

LBP is not chosen as the main algorithm in this application because the prediction of the ending point requires accurate inverse results. This will be proved in the later sections.

\section{2) Iterative Algorithms}

There are many different existing iterative algorithms for ill-posed inverse problems, such as Gauss-Newton, Landweber, and conjugate gradient etc [9, 13, 14]. The common approach for all iterative algorithms is to eliminate errors and converge the result to the true solution through iterative calculations[15]. Iterative algorithms are very effective in static experiments as they can trade off computing time to minimize the error in the result. But they are not adopted in this application because they lower the computing efficiency of online tests.

\section{3) Tikhonov Regularization}

The main objectives of employing regularization in illposed problems are to impose the prior assumptions, which are the reference values in the case of ERT, on the solution and at the same time filter out the high-frequency components of the solution, which correspond to the smallest singular values of the sensitivity matrix[16]. But when there is a significant difference between the prior assumption and the solution, the latter could be seriously distorted after regularization is adopted.

Tikhonov regularization is a widely used algorithm for soft field tomography[11]. The explicit formula pattern is[11]:

$$
\bar{\sigma}_{\alpha}=\left(\bar{J}^{T} \bar{J}+\alpha^{2} I\right)^{-1} \bar{J}^{T} \bar{V}
$$

$\bar{J}^{T}$ stands for the transpose of the sensitivity matrix and $I$ is an identity matrix. $\alpha^{2}$ is known as the regularization parameter, which controls the convergence level of the result. We use $\alpha$ square to imply that it should always be positive.

Tikhonov regularization is chosen to be the basic algorithm in this research because the main objective of monitoring CIP is to find out the ending point which in principle is defined as the point when the last fragment of soil is cleaned. This implies that the solution values would be very close to the reference values, under which condition regularization is most accurate. In the later sections, optimization on conventional Tikhonov regularization will be implemented in order to fulfil comprehensive analytical purposes.

\section{Optimizing Tikhonov Regularization}

\section{1) Basic Principle}

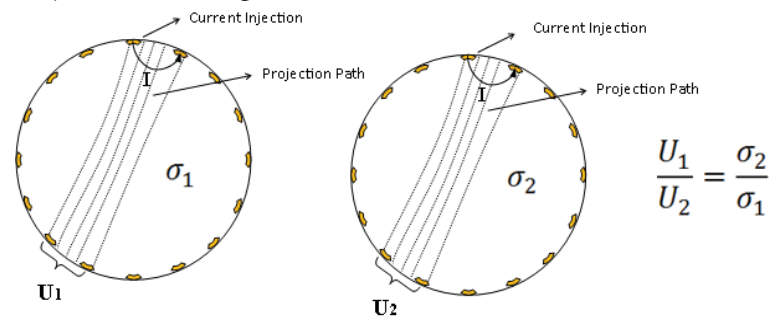

Fig. 1. Relationship between measured boundary voltage and material conductivity under same projection

Two ERT planes with same dimensions and structure are filled respectively by two uniform conductive liquids of different conductivities $\sigma_{1}$ and $\sigma_{2}$ (Figure 1 ). The projection 
paths under the same electrode pairs in the two planes have the same shape. Hence, with fixed injection current on the transmitter electrode pairs, the ratio of the measured boundary voltage across a receiver electrode pair in plane 1 to that of the same positioned pair in plane 2 will equal to the ratio of the liquid conductivity in plane 2 to that of plane1.
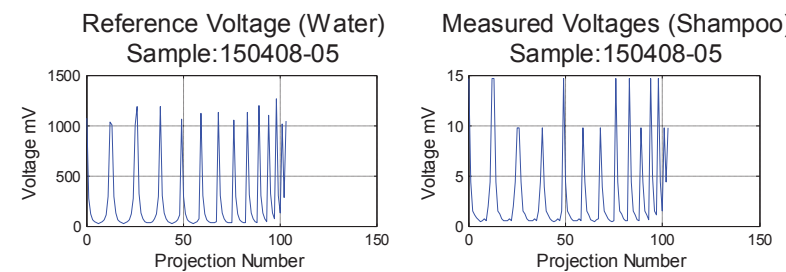

Fig.2. Measured boundary voltages (U curves) comparison between pure water and pure soil.

Similarly for other pairs of electrodes, the complete sets of boundary voltage measurements taken from the two planes, which are also known to be the 'U curves', should also be proportional. This can be proved by Figure 2 . Despite the deviations caused by system noise, the two curves basically stay in the same shape. For all the corresponding voltage values, we also calculated the ratios between them and the result is shown in Table 1. 85 out of 104 ratios stay between $60-80$, which is the actual ratio between the conductivities of the soil and water in our investigation.

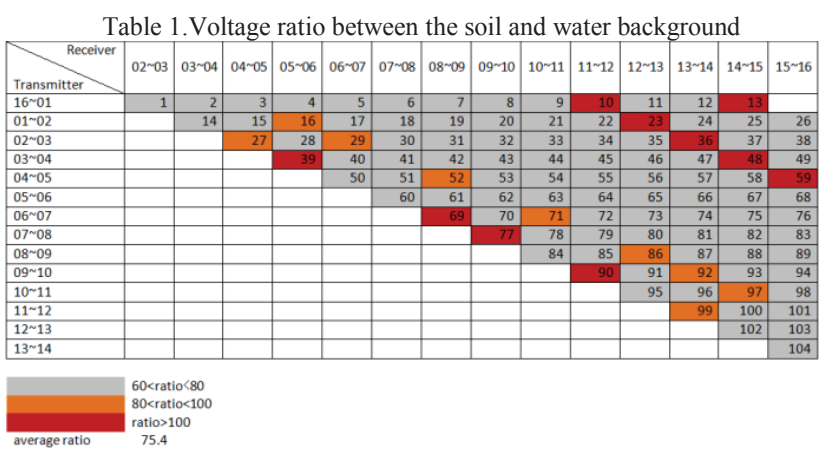

\section{2) Optimization}

The cause of the distortions in preliminary results from Tikhonov regularization is the significant difference between the measured boundary voltages and reference voltages. On the other hand, if the reference voltages can vary dynamically with the change of background material conductivity to approach the level of measured voltages, the distortions can hence be reduced or even eliminated.

The average voltage level can be determined in many ways. The method adopted in this experiment is:

a) Extract the 16 voltage values corresponding to the measurements taken from where the distances between transmitter electrode pair and receiver electrode pair are the smallest and the accuracies are the highest;

b) Eliminate abnormal large values by limiting them to the average level of peak values in the reference voltage; c) The median value of the 16 measured voltages is adopted as the criterion to judge the average voltage level.

Based on above method, the ratio between the average level of the measured voltages and the reference is:

$$
\gamma=\mathrm{V}_{\mathrm{mm}} / \mathrm{V}_{\mathrm{Rm}}
$$

$V_{m m}$ and $V_{R m}$ are the median values of the 16 chosen voltages from measured boundary voltages and reference voltages. Hence, the dynamic reference corresponding to each frame is:

$$
\overline{V_{R}^{*}}=\gamma \cdot \overline{V_{R}}
$$

So the formula of optimized Tikhonov regularization with dynamic reference can be derived as:

$$
\begin{aligned}
& \bar{\sigma}_{\alpha}^{*}=\left(\bar{J}^{T} \bar{J}+\alpha^{2} I\right)^{-1} \bar{J}^{T} \overline{V^{*}} \\
& \bar{\sigma}_{\alpha}^{*}=\left[\frac{\Delta \sigma_{1}}{\gamma \cdot \sigma_{R}}, \frac{\Delta \sigma_{2}}{\gamma \cdot \sigma_{R}} \cdots \frac{\Delta \sigma_{w}}{\gamma \cdot \sigma_{R}}\right]^{-1} \\
& \overline{V^{*}}=\left[\frac{\Delta V_{1}}{V_{R 1}^{*}}, \frac{\Delta V_{2}}{V_{R 2}^{*}} \cdots \frac{\Delta V_{p}}{V_{R p}^{*}}\right]^{-1}
\end{aligned}
$$

\section{EXPERIMENTS AND RESULTS}

\section{A. Instruments and Procedures}

\section{1) Instruments}

The lab CIP circuit is shown in Figure 3.The left end of the testing area is connected to the soil tank and water tap through a three-way valve. In the current study, a nonNewtonian, shear thinning personal care product was used as the soil. The right end leads to the drain with a valve. There are 2 removable test areas into which transparent pipes with different geometry can be installed.

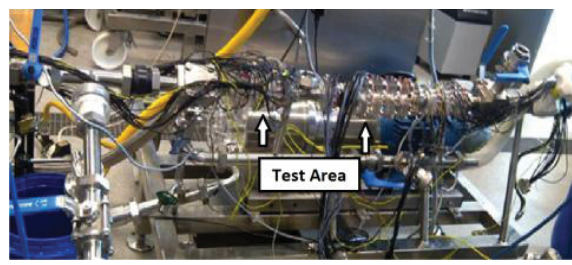

Fig.3.Simulated CIP circuit

In the present research, a ' $\mathrm{T}$-shape' pipe shown in Figure 4 is installed in one of the test areas and the other test area is placed with a normal straight pipe. A total of four ERT planes, each with 16 electrodes, are installed in the test pipe. Planes 1 to 3 are in the straight pipe section and plane 4 is in the sealed concave bottom(Figure 4), which in principle should be the most difficult one to be cleaned. Electrodes on the planes are connected to a commercial ERT system in order to fulfill the measurements.

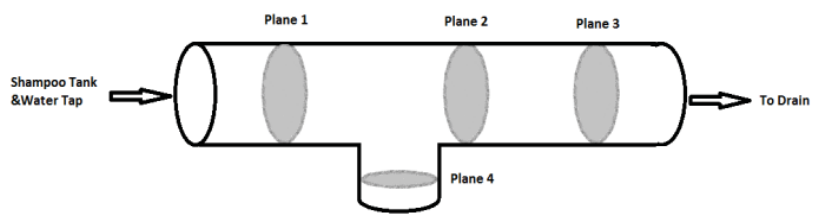

Fig.4. 'T-shape' pipe with the 4 ERT planes installed 


\section{2) Procedures}

The procedure of the simulated test can be concluded to steps as follow:

Step 1: Switch the three-way valve to the branch connected to water tap and fully fill the test area with tap water. Switch on the ERT system to start current injection and voltage measurement. The measured voltage values within this step are considered to be the original reference values.

Step 2: Switch off the ERT system and switch the threeway valve to the soil tank. Fill the test area with the soil. Restart the ERT measurements.

Step 3: Switch the valve to water tap and start the CIP process.

Step 4: Keep measuring until the test area is visually fully cleaned.

So the components being measured under each step are water, soil, the mixture of soil and water, water.

\section{B. Analytical Principles}

The measurement strategy in this experiment is reciprocal adjacent strategy. A total of 104 boundary voltage measurements were taken in each ERT frame.

The average conductivity of the soil is approximately $5 \mathrm{~S} / \mathrm{m}$ while that of tap water is around $0.06 \mathrm{~S} / \mathrm{m}$. Therefore the maximum conductivity value of each ERT frame, i.e. the maximum pixel value in each reconstructed image is extracted independently as the criterion of judging the remaining presence of the soil. The ending point is reached when the maximum pixel value falls back to the reference level. Also, the average conductivity value in each frame is calculated to indicate the overall conductivity level in the steps described in the experimental procedures.

\section{Preliminary Results}

366 frames of data were acquired for each ERT plane during the CIP process under the water flow rate of $5400 \mathrm{~L} / \mathrm{h}$ and with the injection current of $5.11 \mathrm{~mA}$. The ending point for the ERT plane located in the $\mathrm{T}$ junction pipe, where the most difficult point in the pipe is fully cleaned, is shown to be around frame 340 .

1) Inverse Solutions with $L B P$

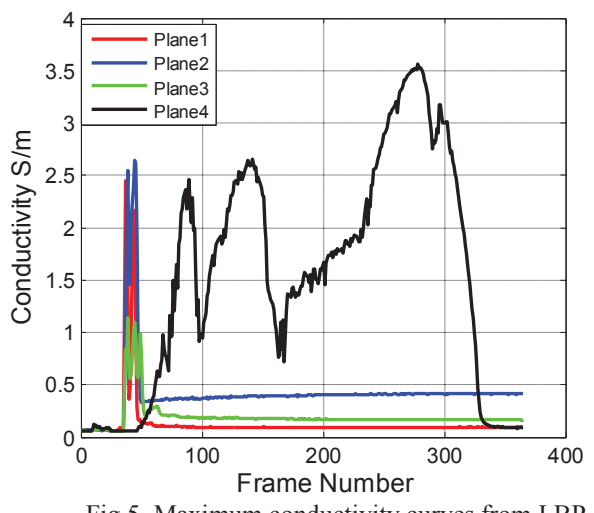

Fig.5. Maximum conductivity curves from LBP

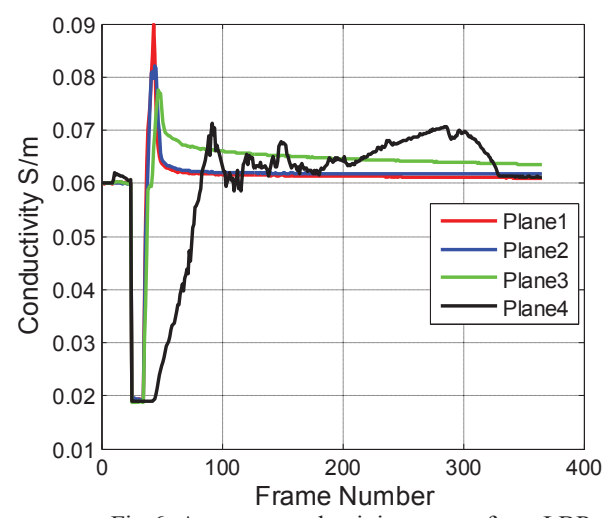

Fig.6. Average conductivity curves from LBP

The inverse solutions generated from LBP are shown in Figures 5 and 6.

The maximum conductivity curves in Figure 5 are capable of indicating the ending point of each plane. However, the conductivity values of plane 2 and plane 3 slightly exceed the actual conductivity of water $(0.06 \mathrm{~S} / \mathrm{m})$ when they are fully cleaned. All the values are lower than the actual soil conductivity $(5 \mathrm{~S} / \mathrm{m})$ to some extent, even during the period when the testing area is fully filled with the soil.

The average conductivity curves of plane 4 drift randomly above and beneath the reference conductivity value in a small range after frame 50 . In addition, at frame 25 when the background material in testing area suddenly changes from water to the soil, the average conductivity value of all 4 planes jump below the reference value, which does not match the real case. Thus they cannot reflect the actual procedures of the experiment.

The results have proved that LBP is not suitable for the monitoring of CIP due to the lack of accuracy.

2) Inverse Solutions with Tikhonov Regularization

The inverse solutions generated from Tikhonov regularization are shown below.

The maximum and average conductivity values in those figures are converged to a much smaller range near reference conductivity value comparing to those of LBP while the curves are spikier under the narrowed axis scale. But the accuracy of the solutions near and after ending point is significantly improved. The maximum conductivity values after ending point of all 4 planes stay steady at the reference level $(0.06 \mathrm{~S} / \mathrm{m})$ without drifting, which enables the position of ending point to be identified clearly. As for the average conductivity curves, they are also very spiky and vibrate within a small range before the ending points. Similar to the case of LBP, they cannot represent the actual procedures either.

The performance can be explained by the convergent nature of Tikhonov regularization. When the conductivity of the target to be tested is significantly different from the reference value, the result will be converged and distorted. In this experiment, from frame 25 when the background material changes from water to soil, up to frame 250 when 
the overall conductivity level drops near the reference level, the inverse solutions would become more and more accurate.

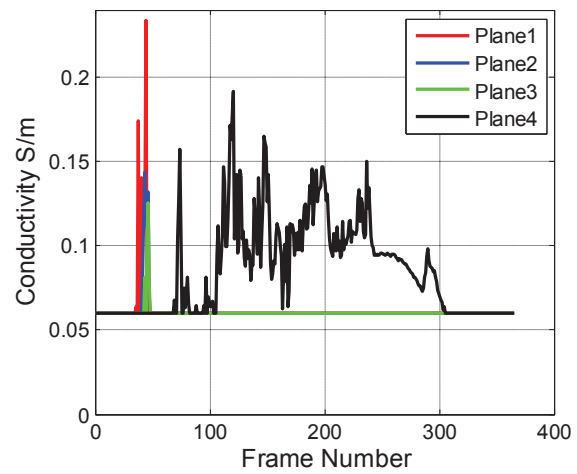

Fig.7. Maximum conductivity curves from Tikhonov regularization

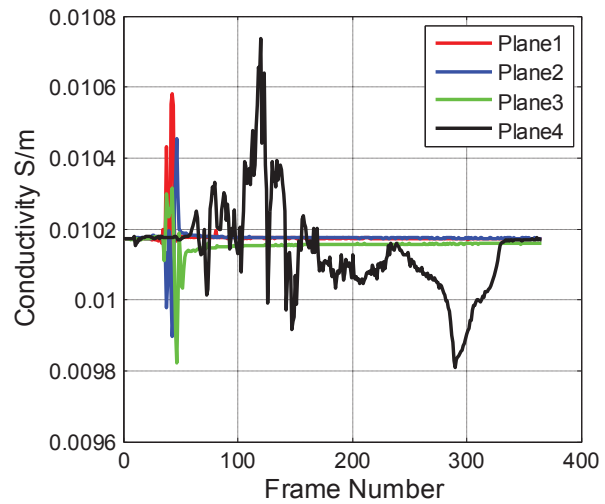

Fig.8. Average conductivity curves from Tikhonov regularization Hence, the objective of locating the accurate ending point is accomplished. But in order to achieve comprehensive analysis throughout the CIP process, optimization needs to be applied to the conventional Tikhonov regularization in order to overcome the distortions in the average conductivity plot at earlier stages of the process.

\section{Optimized Results}

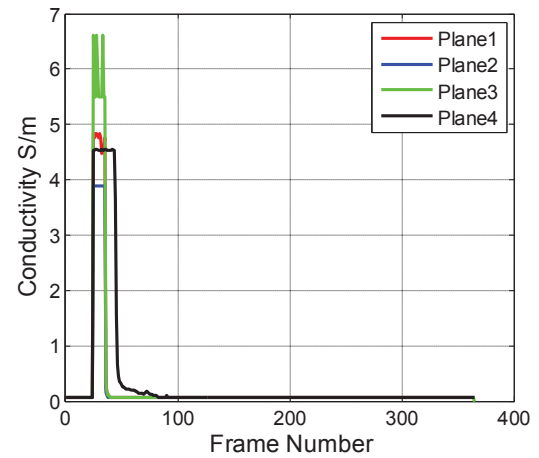

Fig.9. Average conductivity values from optimized algorithm

Figure 9 shows the average conductivity values in each frame. It can be clearly seen in the plot that from frame 0 to around frame 25 , the pipe is filled with water and the average conductivity stays at reference level. Then the pipe is filled by the soil with the conductivity ranging from 4 to 6 $\mathrm{S} / \mathrm{m}$. The CIP process starts from around frame 40 . The majority of the soil in straight pipe section is flushed away rapidly. The optimized ERT results indicate that plane 1 to 3 are cleaned around frame 40.As for plane 4, the majority of the soil still remains even after plane 1 to 3 are already fully cleaned. Then the amount of soil starts to drop significantly from frame 50 until the plane is totally cleaned. In this way, the average conductivity curves fully illustrate the whole process, which is an important enhancement compared to conventional algorithms. This enables researchers to analyze earlier stages of CIP.

The maximum conductivity curves are shown in Figure 10 and 11. Due to system noise and the ill-conditioned nature of inverse problem, the maximum conductivity values are slightly higher and spikier than the average conductivity values. This is not critical as the main objective of plotting the maximum conductivity curve is to locate the ending point. With the scale of $\mathrm{Y}$ axis narrowed to $0 \sim 0.1$ $\mathrm{S} / \mathrm{m}$, the ending point of all 4 planes can be clearly identified.

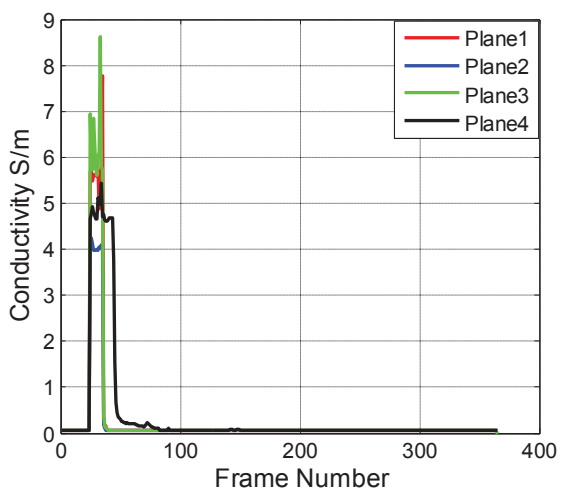

Fig.10. Maximum conductivity curves from optimized algorithm

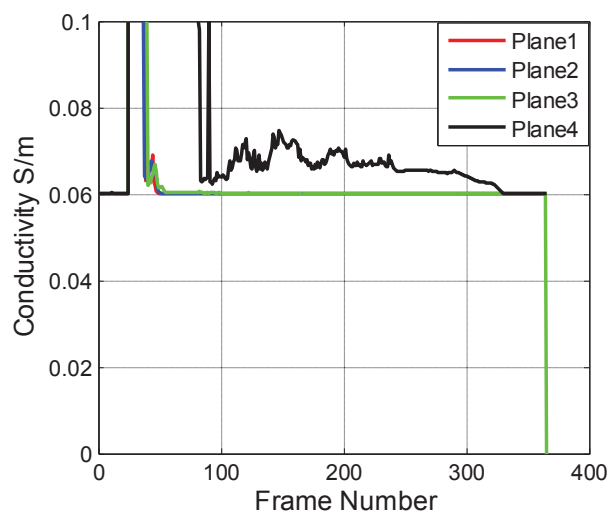

Fig.11. Maximum conductivity curve in smaller scale

Images are also reconstructed from the optimized results (Figure 12). This provides a more intuitive way to inspect what actually happens during the CIP process and to locate the most difficult part to be cleaned on the periphery of the plane. 


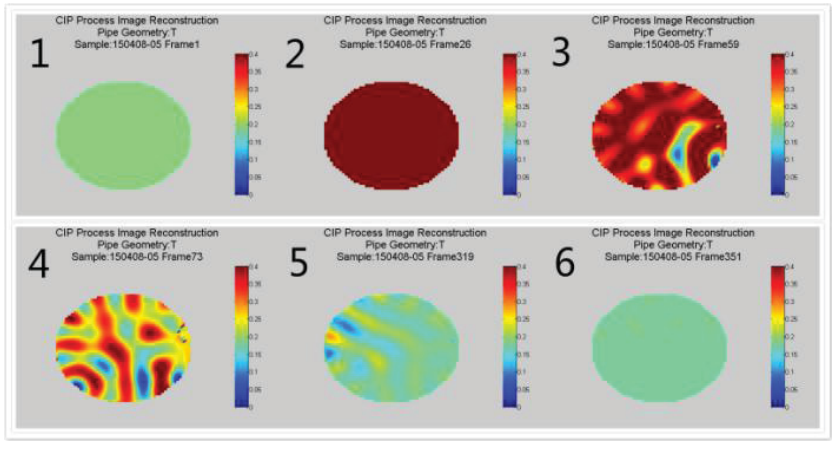

Fig.12. Reconstructed images from optimized results

\section{CONCLUSIONS}

The present paper proposes a new approach of monitoring and analyzing the Clean-In-Place process with Electrical Resistance Tomography. An optimized algorithm with dynamic reference based on Tikhonov regularization is adopted in the inverse calculation. The dynamic reference is generated by configuring the average level of measured boundary voltages. The optimized result shows significant advantages against conventional LBP and Tikhonov regularization as it can precisely locate the ending point of the whole process and it overcomes the distortions when the conductivity of background material varies in a wide range. The sharpness and reliability of the reconstructed images are also improved.

However, some drawbacks still exist in the optimized algorithm as the situation of mixed liquids is not considered in the basic principle. In fact when the plane is filled with equal volume of incompletely mixed liquids of different conductivities, the generated corresponding reference will totally distort the result as it is significantly different from the both components. But as in this experiment, the majority of mixture or mixed liquid is flushed away rapidly because of the high flow rate and the conductivity distribution within this relatively short period is not the main objective, the drawback can thus be neglected under this condition. If the dynamic reference optimization needs to be adopted in other applications, this drawback should be considered in prior.

\section{ACKNOWLEDGEMENTS}

The authors wish to thank Dr. Ruozhou Hou at the School of Chemical Engineering and Analytical Science, University of Manchester, for valuable discussions and provision of the CIP experimental data.

\section{REFERENCE}

1. J. Mendes, A.P., L.F. Melo, Monitoring cleaningin-place of shampoo films using nanovibration technology. Sensors and Actuators, 2009. B 136: p. 376-382.
2. Schöning M J, B.D., Rolka D, et al., CIP (cleaning-in-place) suitable "non-glass" $p H$ sensor based on a Ta 2 O 5-gate EIS structure. Sensors and Actuators, 2005. 111(B): p. 423-429.

3. T.R. Hay, J.L.R., Fouling detection in the food industry using ultrasonic guided waves. Food Control, 2003. 14: p. 481-488.

4. K.R. Lohr, J.L.R., Ultrasonic guided wave and acoustic impact methods for pipe fouling detection. Journal of Food Engineering, 2003. 56: p. 315-324.

5. M.S.Beck, Process Tomography: Principles, Techniques and Applications. 1995: ButterworthHeinemann Ltd.

6. Scott, D.M., Process Imaging For Automatic Control. 2005: Taylor\&Francis Group.

7. Borcea, L., Electrical impedance tomography. Inverse Problems, 2002. 18: p. R99-R136.

8. Murai, T. and Y. Kagawa, Electrical impedance computed tomography based on a finite element model. Biomedical Engineering, IEEE Transactions on, 1985(3): p. 177-184.

9. Wang, M., Inverse solutions for electrical impedance tomography based on conjugate gradients methods. Measurement Science and Technology, 2001. 13(1): p. 101.

10. Yang, W., et al., An image-reconstruction algorithm based on Landweber's iteration method for electrical-capacitance tomography. Measurement Science and Technology, 1999. 10(11): p. 1065.

11. Holder, D.S., Electrical impedance tomography: methods, history and applications. 2004: CRC Press.

12. Yang, W. and L. Peng, Image reconstruction algorithms for electrical capacitance tomography. Measurement Science and Technology, 2002. 14(1): p. R1.

13. $\mathrm{Li}, \mathrm{Y}$. and $\mathrm{W}$. Yang, Image reconstruction by nonlinear Landweber iteration for complicated distributions. Measurement Science and Technology, 2008. 19(9): p. 094014.

14. Schweiger, M., S.R. Arridge, and I. Nissila, GaussNewton method for image reconstruction in diffuse optical tomography. Phys Med Biol, 2005. 50(10): p. 2365-86.

15. Yorkey, T.J., J.G. Webster, and W.J. Tompkins, Comparing reconstruction algorithms for electrical impedance tomography. Biomedical Engineering, IEEE Transactions on, 1987(11): p. 843-852.

16. Polydorides, N., Image reconstruction algorithms for soft-field tomography. 2002, University of Manchester: UMIST. 